\title{
The Effect of Conformal Symmetry on Charged Wormholes
}

\author{
Peter K. F. Kuhfittig \\ Department of Mathematics, Milwaukee School of Engineering, Milwaukee, WI, USA \\ Email:kuhfitti@msoe.edu
}

How to cite this paper: Kuhfittig, P.K.F. (2016) The Effect of Conformal Symmetry on Charged Wormholes. Journal of Appliea Mathematics and Physics, 4, 2117-2125. http://dx.doi.org/10.4236/jamp.2016.412209

Received: November 7, 2016

Accepted: December 2, 2016

Published: December 5, 2016

Copyright $\odot 2016$ by author and Scientific Research Publishing Inc. This work is licensed under the Creative Commons Attribution International License (CC BY 4.0). http://creativecommons.org/licenses/by/4.0/ (c) (i) Open Access

\begin{abstract}
This paper discusses the effect that conformal symmetry can have on a charged wormhole. The analysis yields a physical interpretation of the conformal factor in terms of the electric charge. The rate of change of the conformal factor determines much of the outcome, which ranges from having no solution to wormholes having either one or two throats.
\end{abstract}

\section{Keywords \\ Charged Wormholes, Conformal Symmetry}

\section{Introduction}

Wormholes are handles or tunnels in spacetime connecting different regions of our Universe or different universes altogether. That wormholes could be actual physical structures suitable for interstellar travel was first proposed by Morris and Thorne [1]. Such wormholes can be described by the static and spherically symmetric line element

$$
\mathrm{d} s^{2}=-\mathrm{e}^{2 \Phi(r)} \mathrm{d} t^{2}+\frac{\mathrm{d} r^{2}}{1-b(r) / r}+r^{2}\left(\mathrm{~d} \theta^{2}+\sin ^{2} \theta \mathrm{d} \phi^{2}\right),
$$

using units in which $c=G=1$. Here $\Phi=\Phi(r)$ is called the redshift function, which must be everywhere finite to avoid an event horizon. The function $b=b(r)$ is called the shape function since it determines the spatial shape of the wormhole when viewed, for example, in an embedding diagram [1]. The spherical surface $r=r_{0}$ is the throat of the wormhole. Here $b(r)$ must satisfy the following conditions: $b\left(r_{0}\right)=r_{0}$, $b(r)<r$ for $r>r_{0}$, and $b^{\prime}\left(r_{0}\right) \leq 1$, usually called the flare-out condition. For Morris-Thorne wormholes, this condition can only be satisfied by violating the null energy condition, requiring the use of "exotic matter." Since $b^{\prime}(r)$ is proportional to the den- 
sity in the Einstein field equations, we ordinarily require that $b^{\prime}(r)>0$.

In this paper, we study the effect of conformal symmetry on wormholes that have an electric charge. More precisely, we assume the existence of a conformal Killing vector $\xi$ defined by the action of $\mathcal{L}_{\xi}$ on the metric tensor:

$$
\mathcal{L}_{\xi} g_{\mu v}=\psi(r) g_{\mu v},
$$

where $\mathcal{L}_{\xi}$ is the Lie derivative operator and $\psi(r)$ is the conformal factor. Charged wormholes were first proposed by Kim and Lee [2]. Compatibility of such wormholes with quantum field theory is discussed in Ref. [3].

In addition to studying its effect on a charged wormhole, we obtain a physical interpretation of the conformal factor in terms of the electric charge. The combination of electric charge and conformal symmetry results in a wormhole model that may actually have two throats.

\section{Conformal Killing Vectors}

As noted in the Introduction, we assume that our static spherically symmetric spacetime admits a one-parameter group of conformal motions, i.e., motions along which the metric tensor remains invariant up to a scale factor. Equivalently, there exist conformal Killing vectors such that

$$
\mathcal{L}_{\xi} g_{\mu \nu}=g_{\eta v} \xi_{; \mu}^{\eta}+g_{\mu \eta} \xi_{; \nu}^{\eta}=\psi(r) g_{\mu \nu},
$$

where the left-hand side is the Lie derivative of the metric tensor and $\psi(r)$ is the conformal factor [4] [5]. The vector $\xi$ generates the conformal symmetry and the metric tensor $g_{\mu v}$ is conformally mapped into itself along $\xi$. As discussed in Refs. [6] [7], this type of symmetry has been used effectively to describe relativistic stellar-type objects, thereby leading to new solutions, as well as new geometric and kinematical insights [8] [9] [10] [11] [12]. Even more significantly, it has also been shown that the Kerr black hole is conformally symmetric [13]. Two earlier studies assumed non-static conformal symmetry [5] [12].

To study the effect of conformal symmetry, it is convenient to use an alternate form of the metric [14] [15]:

$$
\mathrm{d} s^{2}=-\mathrm{e}^{v(r)} \mathrm{d} t^{2}+\mathrm{e}^{\lambda(r)} \mathrm{d} r^{2}+r^{2}\left(\mathrm{~d} \theta^{2}+\sin ^{2} \theta \mathrm{d} \phi^{2}\right) .
$$

Using this form, the Einstein field equations become

$$
\begin{aligned}
& \mathrm{e}^{-\lambda}\left(\frac{\lambda^{\prime}}{r}-\frac{1}{r^{2}}\right)+\frac{1}{r^{2}}=8 \pi \rho, \\
& \mathrm{e}^{-\lambda}\left(\frac{1}{r^{2}}+\frac{v^{\prime}}{r}\right)-\frac{1}{r^{2}}=8 \pi p_{r},
\end{aligned}
$$

and

$$
\frac{1}{2} \mathrm{e}^{-\lambda}\left[\frac{1}{2}\left(v^{\prime}\right)^{2}+v^{\prime \prime}-\frac{1}{2} \lambda^{\prime} v^{\prime}+\frac{1}{r}\left(v^{\prime}-\lambda^{\prime}\right)\right]=8 \pi p_{t} .
$$

To keep the analysis tractable, we follow Herrera and Ponce de León [6] and restrict 
the vector field by requiring that $\xi^{\alpha} U_{\alpha}=0$, where $U_{\alpha}$ is the four-velocity of the perfect fluid distribution, so that fluid flow lines are mapped conformally onto fluid flow lines. The assumption of spherical symmetry then implies that $\xi^{0}=\xi^{2}=\xi^{3}=0$ [6]. Equation (3) now yields the following results:

$$
\begin{aligned}
& \xi^{1} v^{\prime}=\psi, \\
& \xi^{1}=\frac{\psi r}{2},
\end{aligned}
$$

and

$$
\xi^{1} \lambda^{\prime}+2 \xi_{, 1}^{1}=\psi
$$

From Equations (8) and (9), we then obtain $v^{\prime}=2 / r$ and thereby

$$
\mathrm{e}^{v}=c_{1} r^{2}
$$

where $c_{1}$ is an integration constant. Now from Equation (9) we get

$$
\xi_{, 1}^{1}=\frac{1}{2}\left(\psi^{\prime} r+\psi\right) .
$$

Substituting in Equation (10) and using $v^{\prime}=2 / r$, simplification yields

$$
\lambda^{\prime}=-2 \frac{\psi^{\prime}}{\psi} \text {. }
$$

Solving for $\lambda$ produces the final result,

$$
\mathrm{e}^{\lambda}=\left(\frac{c_{2}}{\psi}\right)^{2},
$$

where $c_{2}$ is another integration constant.

The Einstein field equations can be rewritten as follows:

$$
\begin{aligned}
& \frac{1}{r^{2}}\left(1-\frac{\psi^{2}}{c_{2}^{2}}\right)-\frac{\left(\psi^{2}\right)^{\prime}}{c_{2}^{2} r}=8 \pi \rho, \\
& \frac{1}{r^{2}}\left(\frac{3 \psi^{2}}{c_{2}^{2}}-1\right)=8 \pi p_{r},
\end{aligned}
$$

and

$$
\frac{\psi^{2}}{c_{2}^{2} r^{2}}+\frac{\left(\psi^{2}\right)^{\prime}}{c_{2}^{2} r}=8 \pi p_{t} .
$$

It now becomes apparent that $c_{2}$ is merely a scale factor in Equations (12)-(15); so we may assume that $c_{2}=1$. The constant $c_{1}$, on the other hand, will have to be obtained from the junction conditions, the need for which can be seen from Equation (11): since our wormhole spacetime is not asymptotically flat, the wormhole material must be cut off at some $r=a$ and joined to an exterior Schwarzschild solution,

$$
\mathrm{d} s^{2}=-\left(1-\frac{2 M}{r}\right) \mathrm{d} t^{2}+\frac{\mathrm{d} r^{2}}{1-2 M / r}+r^{2}\left(\mathrm{~d} \theta^{2}+\sin ^{2} \theta \mathrm{d} \phi^{2}\right),
$$


so that $\mathrm{e}^{v(a)}=c_{1} a^{2}=1-2 M / a$, whence

$$
c_{1}=\frac{1-2 M / a}{a^{2}}
$$

where $M$ is the mass of the wormhole as seen by a distant observer.

\section{Charged Wormholes}

It was proposed by Kim and Lee [2] that for a wormhole with constant charge $Q$ the Einstein field equations take on the form

$$
G_{\mu \nu}^{(0)}+G_{\mu \nu}^{(1)}=8 \pi\left[T_{\mu \nu}^{(0)}+T_{\mu \nu}^{(1)}\right] .
$$

Given that the usual form is $G_{\mu \nu}^{(0)}=8 \pi T_{\mu \nu}^{(0)}$, the modified form in Equation (18) is obtained by adding the matter term $T_{\mu \nu}^{(1)}$ to the right side and the corresponding back reaction term $G_{\mu \nu}^{(1)}$ to the left side. The proposed metric is

$$
\mathrm{d} s^{2}=-\left(1+\frac{Q^{2}}{r^{2}}\right) \mathrm{d} t^{2}+\left(1-\frac{b(r)}{r}+\frac{Q^{2}}{r^{2}}\right)^{-1} \mathrm{~d} r^{2}+r^{2}\left(\mathrm{~d} \theta^{2}+\sin ^{2} \theta \mathrm{d} \phi^{2}\right) .
$$

Kim and Lee go on to note that with $b \equiv 0$, the wormhole becomes a ReissnerNordström black hole, and if $Q=0$, the spacetime becomes a Morris-Thorne wormhole with a shape function $b=b(r)$ that meets the usual requirements. It therefore became necessary to show that the metric, Equation (19), is a self-consistent solution of the Einstein field equations. The shape function $b=b(r)$ of the Morris-Thorne wormhole is now replaced by the effective shape function

$$
b_{\text {eff }}(r)=b(r)-\frac{Q^{2}}{r} .
$$

The effective shape function also has the usual properties, to be discussed later.

\section{Charged Wormholes with Conformal Symmetry}

In this section we return to the assumption of conformal symmetry mentioned in Section 2. Let us first consider the Kim-Lee model, Equation (19). Then by Equation (11), $\mathrm{e}^{v}=c_{1} r^{2}$, we have, for all $r$,

$$
1+\frac{Q^{2}}{r^{2}}=c_{1} r^{2}
$$

which is impossible. So this model is not compatible with the assumption of conformal symmetry. This difficulty can be overcome, however, by introducing a new differentiable function $S(r)$ to yield the line element

$$
\mathrm{d} s^{2}=-\left(1+S(r)+\frac{Q^{2}}{r^{2}}\right) \mathrm{d} t^{2}+\left(1-\frac{b(r)}{r}+\frac{Q^{2}}{r^{2}}\right)^{-1} \mathrm{~d} r^{2}+r^{2}\left(\mathrm{~d} \theta^{2}+\sin ^{2} \theta \mathrm{d} \phi^{2}\right) .
$$

Evidently,

$$
S(r)=-\left(1+\frac{Q^{2}}{r^{2}}\right)+r^{2} \frac{1-2 M / a}{a^{2}}
$$


by Equation (17). Since $a>r$ on the interval $\left[r_{0}, a\right]$, it follows that $S(r)<0$, while $S^{\prime}(r)>0$.

As already noted, given the effective shape function $b_{\text {eff }}(r)=b(r)-Q^{2} / r$ and the total matter $T^{\text {eff }}=T_{\mu \nu}^{(0)}+T_{\mu \nu}^{(1)}$, the Kim-Lee model yields a self-consistent solution. The inclusion of $S(r)$ has no effect on this conclusion. So our metric, Equation (21), is a valid solution of the Einstein field equations representing a wormhole with an electric charge.

The major objective in this section is to obtain a physical interpretation of the conformal factor $\psi(r)$, as well as the restrictions required to obtain a wormhole. First we recall that for some $r=r_{1}, b\left(r_{1}\right)=r_{1}$ and $b^{\prime}\left(r_{1}\right) \leq 1$. Also, for $r>r_{1}, b(r)<r$. For the new (effective) shape function, $b_{\text {eff }}(r)=b(r)-Q^{2} / r$, we have $b_{\text {eff }}\left(r_{0}\right)=r_{0}$ and $b_{\text {eff }}^{\prime}\left(r_{0}\right) \leq 1$ to meet the flare-out condition. (This implies that $b^{\prime}\left(r_{0}\right) \leq 1-Q^{2} / r_{0}^{2}$.) Since $b=b(r)$ is assumed to be a typical shape function, $b(r)<r$ for $r>r_{1}$, and $b(r)>r$ for $r<r_{1}$. Since $b\left(r_{0}\right)-r_{0}=Q^{2} / r_{0}>0$ by Equation (20), it follows that $r_{0}<r_{1}$ (see Figure 1).

Next, from Equations (4), (12), and (21) (and recalling that $c_{2}=1$ ),

$$
1-\frac{b(r)}{r}+\frac{Q^{2}}{r^{2}}=\psi^{2}(r) \text {. }
$$

Since $b(r) / r=1+Q^{2} / r^{2}-\psi^{2}(r)$, it also follows that

$$
b(r)=r\left(1+\frac{Q^{2}}{r^{2}}-\psi^{2}(r)\right)
$$

and

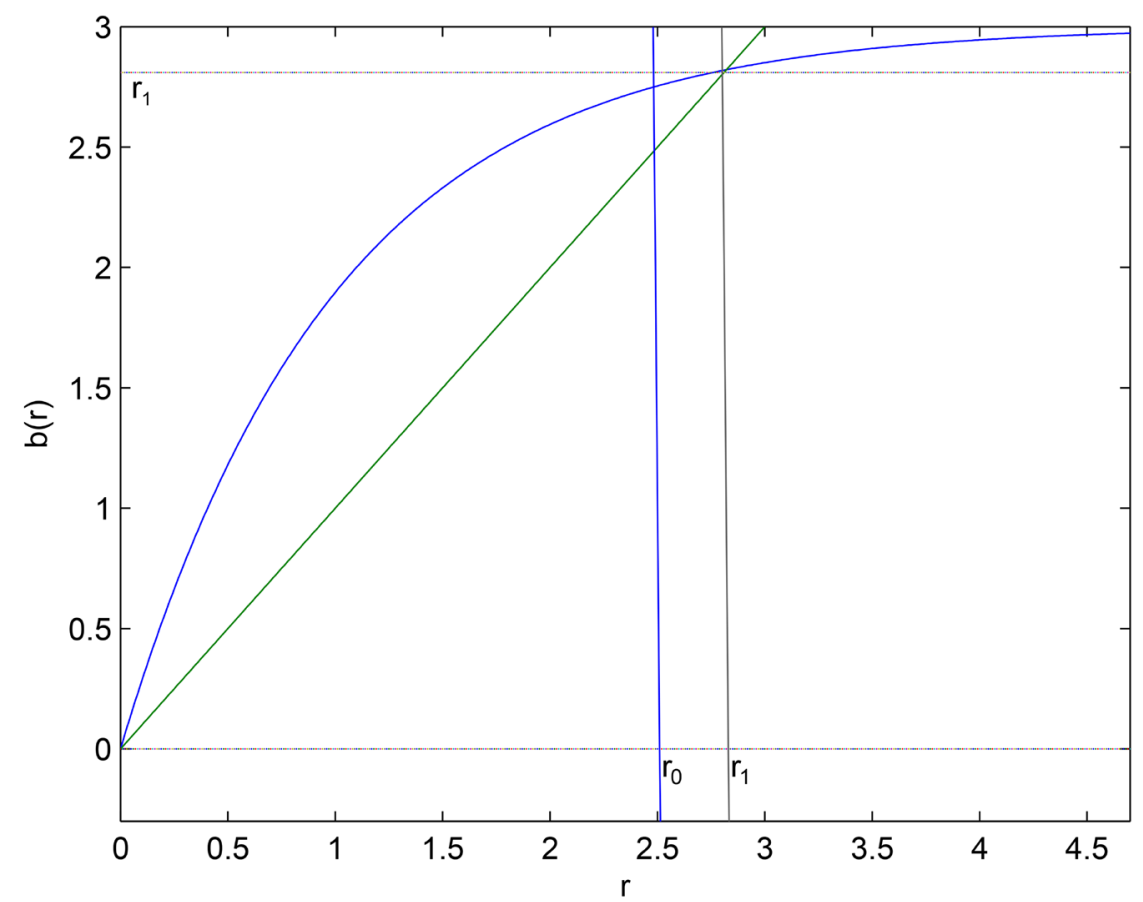

Figure 1. The throat of $b_{\text {eff }}(r)$ at $r=r_{0}$ remains near $r=n$. 


$$
b_{\text {eff }}(r)=r\left(1+\frac{Q^{2}}{r^{2}}-\psi^{2}(r)-\frac{Q^{2}}{r^{2}}\right)=r\left(1-\psi^{2}(r)\right) .
$$

The condition $b_{\text {eff }}\left(r_{0}\right)=r_{0}$ now implies that

$$
\psi^{2}\left(r_{0}\right)=0 .
$$

Also, since $b\left(r_{1}\right)=r_{1}$, we have

$$
1-\frac{b\left(r_{1}\right)}{r_{1}}+\frac{Q^{2}}{r_{1}^{2}}=\psi^{2}\left(r_{1}\right)
$$

and

$$
\psi^{2}\left(r_{1}\right)=\frac{Q^{2}}{r_{1}^{2}}
$$

So Equations (26) and (27) give us a physical interpretation for the conformal factor $\psi(r)$ in terms of the charge $Q$. Additional connections to $Q$ are given below.

Having just learned that $\psi^{2}(r)$ increases to the right of $r=r_{0}$, let us examine the slope of $\psi^{2}(r)$ more closely. First note that from Equation (24) we have

$$
b^{\prime}(r)=1-\psi^{2}(r)-\left.r \frac{\mathrm{d}}{\mathrm{d} r} \psi^{2}(r)\right|_{r=r_{0}}=1-r_{0} \frac{\mathrm{d}}{\mathrm{d} r} \psi^{2}\left(r_{0}\right) .
$$

From the inequality $0<b_{\text {eff }}^{\prime}\left(r_{0}\right) \leq 1$, we get

$$
0<b^{\prime}\left(r_{0}\right)+\frac{Q^{2}}{r_{0}^{2}}=1-r_{0} \frac{\mathrm{d}}{\mathrm{d} r} \psi^{2}\left(r_{0}\right)+\frac{Q^{2}}{r_{0}^{2}} \leq 1 .
$$

Solving, we obtain the inequality

$$
\frac{Q^{2}}{r_{0}^{3}} \leq \frac{\mathrm{d}}{\mathrm{d} r} \psi^{2}\left(r_{0}\right)<\frac{1}{r_{0}}+\frac{Q^{2}}{r_{0}^{3}} .
$$

Similarly, since $\psi^{2}\left(r_{1}\right)=Q^{2} / r_{1}^{2}$ and $0<b^{\prime}\left(r_{1}\right) \leq 1$, we have

$$
0<1-\frac{Q^{2}}{r_{1}^{2}}-r_{1} \frac{\mathrm{d}}{\mathrm{d} r} \psi^{2}\left(r_{1}\right) \leq 1 .
$$

Solving, we obtain the second inequality:

$$
-\frac{Q^{2}}{r_{1}^{3}} \leq \frac{\mathrm{d}}{\mathrm{d} r} \psi^{2}\left(r_{1}\right)<\frac{1}{r_{1}}-\frac{Q^{2}}{r_{1}^{3}} .
$$

Our final task is to check the violation of the null energy condition (NEC) required to hold the wormhole open. Recall that the NEC states that given the stress-energy tensor $T_{\alpha \beta}, T_{\alpha \beta} \mu^{\alpha} \mu^{\beta} \geq 0$ for all null vectors. So we obtain from the null vector $(1,1,0,0)$ that $\rho+p_{r}<0$ whenever the condition is violated. By Ref. [1], this violation is equivalent to the condition

$$
\frac{b^{\prime}\left(r_{0}\right)-b\left(r_{0}\right) / r_{0}}{2\left[b\left(r_{0}\right)\right]^{2}}<0
$$

for a generic shape function. For $b_{\text {eff }}(r)$, we already know that the last inequality 
holds whenever $\frac{\mathrm{d}}{\mathrm{d} r} \psi^{2}\left(r_{0}\right) \geq Q^{2} / r_{0}^{3}$ by inequality (29). By Equation (23), $\psi^{2}(r)$ is associated with $b_{\text {eff }}$; so we can use the field equations, Equations (13) and (14), to determine

$$
\left.8 \pi\left(\rho+p_{r}\right)\right|_{r=r_{0}}=-\frac{1}{r_{0}} \frac{\mathrm{d}}{\mathrm{d} r} \psi^{2}\left(r_{0}\right)
$$

since $\psi^{2}\left(r_{0}\right)=0$. Near the throat $r=r_{0}, \psi^{2}(r)$ is rising, so that the NEC is indeed violated at and near $r=r_{0}$.

For $r=r_{1}$, the throat of $b=b(r)$, we can not use Equations (13) and (14), since the null vectors are not the same. Moreover, we can infer from Equation (28) that

$$
0<\psi^{2}(r)+r \frac{\mathrm{d}}{\mathrm{d} r} \psi^{2}(r)<1
$$

showing that $\psi^{2}(r)$ cannot keep increasing indefinitely. In fact, given that $\frac{\mathrm{d}}{\mathrm{d} r} \psi^{2}\left(r_{1}\right) \geq-Q^{2} / r_{1}^{3}, \psi^{2}(r)$ could already be decreasing at $r=r_{1}$. We therefore have to require that $r=r_{1}$ be close enough to $r=r_{0}$ so that

$$
\frac{\mathrm{d}}{\mathrm{d} r} \psi^{2}\left(r_{1}\right) \geq 0
$$

(Since $Q^{2}$ is small in geometrized units, $r_{1}$ is close to $r_{0}$ to begin with.) As a result, if the NEC is violated at $r=r_{0}$, it is also violated at $r=r_{1}$.

\section{An Analogue of the Kerr-Newman Black Hole}

In thia section we study the various conditions under which the NEC is violated. So let us restate Inequality (29) and the modified Inequality (30):

$$
\frac{Q^{2}}{r_{0}^{3}} \leq \frac{\mathrm{d}}{\mathrm{d} r} \psi^{2}\left(r_{0}\right)<\frac{1}{r_{0}}+\frac{Q^{2}}{r_{0}^{3}}
$$

and

$$
0 \leq \frac{\mathrm{d}}{\mathrm{d} r} \psi^{2}\left(r_{1}\right)<\frac{1}{r_{1}}-\frac{Q^{2}}{r_{1}^{3}}
$$

From

$$
0<\frac{Q^{2}}{r_{0}^{3}}<\frac{1}{r_{1}}-\frac{Q^{2}}{r_{1}^{3}}<\frac{1}{r_{0}}+\frac{Q^{2}}{r_{0}^{3}},
$$

we obtain the following half-open intervals:

$$
\begin{aligned}
& \text { Interval I: }\left[0, \frac{Q^{2}}{r_{0}^{3}}\right), \\
& \text { Interval II: }\left[\frac{Q^{2}}{r_{0}^{3}}, \frac{1}{r_{1}}-\frac{Q^{2}}{r_{1}^{3}}\right),
\end{aligned}
$$

and 


$$
\text { Interval III: }\left[\frac{1}{r_{1}}-\frac{Q^{2}}{r_{1}^{3}}, \frac{1}{r_{0}}+\frac{Q^{2}}{r_{0}^{3}}\right) \text {. }
$$

We now observe that whenever $\frac{\mathrm{d}}{\mathrm{d} r} \psi^{2}(r)$ is in Interval I, the NEC is violated at $r=r_{1}$, but not at $r=r_{0}$. (The reason is that $\frac{\mathrm{d}}{\mathrm{d} r} \psi^{2}\left(r_{1}\right)$ now satisfies Inequality (35), but $\frac{\mathrm{d}}{\mathrm{d} r} \psi^{2}\left(r_{0}\right)$ does not satisfy Inequality (34).) So only $b(r)$ has a legitimate throat. If $\frac{\mathrm{d}}{\mathrm{d} r} \psi^{2}(r)$ is in Interval III, the NEC is violated at $r=r_{0}$, but not at $r=r_{1}$. (Here $\frac{\mathrm{d}}{\mathrm{d} r} \psi^{2}\left(r_{0}\right)$ satisfies Inequality (34), but $\frac{\mathrm{d}}{\mathrm{d} r} \psi^{2}\left(r_{1}\right)$ does not satisfy Inequality (35).) So only $b_{\text {eff }}(r)$ has a legitimate throat. Finally, if $\frac{\mathrm{d}}{\mathrm{d} r} \psi^{2}(r)$ is in Interval II, the NEC is violated at both $r_{0}$ and $r_{1}$. So given the right conditions, our wormhole can have two throats.

Now recall that the event horizon of a black hole is often viewed as the analogue of the throat of the wormhole. In fact, according to Hayward [16], if enough negative energy is injected into a black hole, it may become a traversable wormhole; the event horizon becomes the throat. From this perspective, our wormhole can be viewed as the natural analogue of the Kerr-Newman black hole: this type of black hole also has two surfaces that are characterized by coordinate singularities.

Remark: The existence of two throats invites the following speculation: a variation on the Kim-Lee model is

$$
\mathrm{d} s^{2}=-\mathrm{e}^{\Phi(r, Q)} \mathrm{d} t^{2}+\left(1-\frac{b(r)}{r}+\frac{Q^{2}}{r^{2}}\right)^{-1} \mathrm{~d} r^{2}+r^{2}\left(\mathrm{~d} \theta^{2}+\sin ^{2} \theta \mathrm{d} \phi^{2}\right) .
$$

Unlike our earlier model, this metric can lead to an event horizon. In particular, suppose

$$
\mathrm{e}^{\Phi(r, Q)}=\mathrm{e}^{-Q^{2} /\left(r-r_{2}\right)^{2}},
$$

where $r_{0}<r_{2}<r_{1}$. Since Inequalities (34) and (35) still hold, this model suggests that it is in principle possible to pass through the throat at $r=r_{1}$ and return via the throat at $r=r_{0}$, not only bypassing the event horizon of the black hole, but allowing a return trip.

\section{Conclusions}

This paper discusses the effect that conformal symmetry can have on a charged wormhole. Conversely, the physical requirements are seen to place severe constraints on the wormhole geometry.

The analysis yields a physical interpretation of the conformal factor $\psi(r)$ in terms of the charge $Q$. Moreover, the outcome is heavily dependent on $\psi^{2}(r)$ and $\frac{\mathrm{d}}{\mathrm{d} r} \psi^{2}(r)$ 
and ranges from having no solution to wormholes having two throats. The latter case can be viewed as the analogue of the Kerr-Newman black hole.

\section{References}

[1] Morris, M.S. and Thorne, K.S. (1988) Wormholes in Spacetime and Their Use for Interstellar Travel: A Tool for Teaching General Relativity. American Journal of Physics, 56, 395412. https://doi.org/10.1119/1.15620

[2] Kim, S.-W. and Lee, H. (2001) Stability of the Regular Hayward Thin-Shell Wormholes. Physical Review D, 63, Article ID: 064014.

[3] Kuhfittig, P.K.F. (2011) On the Feasibility of Charged Wormholes. Central European Journal of Physics, 9, 1144-1150.

[4] Maartens, R. and Mellin, C.M. (1996) Inhomogeneous Universes in Observational Coordinates. Classical and Quantum Gravity, 13, Article ID: 1571. https://doi.org/10.1088/0264-9381/13/6/030

[5] Böhmer, C.G., Harko, T. and Lobo, F.S.V. (2007) Conformal Symmetry Traversable Wormholes. Physical Review D, 76, Article ID: 084014. https://doi.org/10.1103/PhysRevD.76.084014

[6] Herrera, L. and Ponce de León, J. (1985) Perfect Fluid Spheres Admitting a One-Parameter Group of Conformal Motions. Journal of Mathematical Physics, 26, 778-784. https://doi.org/10.1063/1.526567

[7] Herrera, L. and Ponce de León, J. (1985) Anisotropic Spheres Admitting a One-Parameter Group of Conformal Motions. Journal of Mathematical Physics, 26, 2018-2023. https://doi.org/10.1063/1.526872

[8] Mars, M. and Senovilla, J.M.M. (1993) Axial Symmetry and Conformal Killing Vectors. Classical and Quantum Gravity, 10, 1633-1647. https://doi.org/10.1088/0264-9381/10/8/020

[9] Ray, S., Usmani, A.A., Rahaman, F., Kalam, M. and Chakraborty K. (2008) Electromagnetic Mass Model Admitting Conformal Motion. Indian Journal of Physics, 82, 1191.

[10] Rahaman, F., Jamil, M., Kalam, M., Chakraborty, K. and Ghosh, A. (2010) On Role of Pressure Anisotropy for Relativistic Stars Admitting Conformal Motion. Astrophysics and Space Science 325, 137-147. https://doi.org/10.1007/s10509-009-0167-7

[11] Rahaman, F., Ray, S., Karar, I., Fatima, H.I., Bhowmick, S. and Ghosh, G.K. arXiv:1211.1228 [gr-qc]

[12] Böhmer, C.G., Harko, T. and Lobo, F.S.N. (2008) Wormhole Geometries with Conformal Motions. Classical and Quantum Gravity, 25, Article ID: 075016. https://doi.org/10.1088/0264-9381/25/7/075016

[13] Castro, A., Maloney, A. and Strominger, A. (2010) Hidden Conformal Symmetry of the Kerr Black Hole. Physical Review D, 82, Article ID: 024008. https://doi.org/10.1103/PhysRevD.82.024008

[14] Rahaman, F., Ray, S., Khadekar, G.S., Kuhfittig, P.K.F. and Karar, I. (2015) Noncommutative Geometry Inspired Wormholes with Conformal Motion. International Journal of Theoretical Physics, 54, 699-709. https://doi.org/10.1007/s10773-014-2262-y

[15] Kuhfittig, P.K.F. (2016) Stable Wormholes on a Noncommutative-Geometry Background Admitting a One-Parameter Group of Conformal Motions. Indian Journal of Physics, 90, 837-842. https://doi.org/10.1007/s12648-015-0812-7

[16] Hayward, S.A. arXiv:gr-qc/0203051 
Submit or recommend next manuscript to SCIRP and we will provide best service for you:

Accepting pre-submission inquiries through Email, Facebook, LinkedIn, Twitter, etc. A wide selection of journals (inclusive of 9 subjects, more than 200 journals)

Providing 24-hour high-quality service

User-friendly online submission system

Fair and swift peer-review system

Efficient typesetting and proofreading procedure

Display of the result of downloads and visits, as well as the number of cited articles

Maximum dissemination of your research work

Submit your manuscript at: http://papersubmission.scirp.org/

Or contact jamp@scirp.org 